\title{
Networks, Fields and Organizations: Micro-Dynamics, Scale and Cohesive Embeddings*
}

\author{
DOUGLAS R. WHITE \\ Research Focus Group in Social Dynamics and Evolution, Institute for Mathematical Behavioral Sciences, \\ University of California at Irvine, Irvine CA 92697, USA
}

email:drwhite@uci.edu

JASON OWEN-SMITH

Sociology and Organization Studies, University of Michigan, Ann Arbor, MI 48104-2590, USA

email:jdos@umich.edu

JAMES MOODY

Sociology, Ohio State University, Columbus, OH 43215, USA

email:moody.77@sociology.osu.edu

WALTER W. POWELL

Education, Sociology, and Graduate School of Business, Stanford University, Stanford, CA 94305-3084, USA

email: woodyp@stanford.edu

\begin{abstract}
Social action is situated in fields that are simultaneously composed of interpersonal ties and relations among organizations, which are both usefully characterized as social networks. We introduce a novel approach to distinguishing different network macro-structures in terms of cohesive subsets and their overlaps. We develop a vocabulary that relates different forms of network cohesion to field properties as opposed to organizational constraints on ties and structures. We illustrate differences in probabilistic attachment processes in network evolution that link on the one hand to organizational constraints versus field properties and to cohesive network topologies on the other. This allows us to identify a set of important new micro-macro linkages between local behavior in networks and global network properties. The analytic strategy thus puts in place a methodology for Predictive Social Cohesion theory to be developed and tested in the context of informal and formal organizations and organizational fields. We also show how organizations and fields combine at different scales of cohesive depth and cohesive breadth. Operational measures and results are illustrated for three organizational examples, and analysis of these cases suggests that different structures of cohesive subsets and overlaps may be predictive in organizational contexts and similarly for the larger fields in which they are embedded. Useful predictions may also be based on feedback from level of cohesion in the larger field back to organizations, conditioned on the level of multiconnectivity to the field.
\end{abstract}

Keywords: social cohesion, complex networks, organizational fields, scaling and attachment, macro-micro linkages

*This work was supported by NSF grants \#BCS-9978282 to White (on Predictive Social Cohesion Theory; see http://eclectic.ss.uci.edu/ drwhite/links2pdf.htm) and \# ITR/SOC-0080860 (to Moody), and a Hewlett Foundation grant (to Powell). 


\section{Introduction}

Although many authors employ metaphors that highlight transitions from networks to organizations, such distinctions can miss the point that organizations are composed of networks. These networks operate at multiple levels within and across organizations, and include networks of ideas and classifications, networks of overlapping groups, interpersonal networks, contractual networks, or production chains (to list but a few). The idea that organizations emerge within social fields constituted by networks is also conveyed by metaphors such as "networks into organizations" or "markets from networks" (White, 2002). A littleconsidered implication of the latter conceptions of organizational emergence is the possibility that purely relational data may embody some of the distinctive features of organizations and fields. In principle, observations on network ties may provide a route for the a priori identification of organizations and fields.

Organizational theory has embraced network concepts but in a potentially limiting manner. Our aim here is to situate network ideas, particularly those on social embeddedness, within the wider concept of an organizational field (Bourdieu, 1992; DiMaggio and Powell, 1983). This effort will help frame the relevance of networks to organization theory and create a larger frame in which to theorize the interface between organizations and their external environments. Our core insight is that both organizations and fields emerge from relational networks, and that different micro-behavioral or attachment processes, once established, structure those networks in predictable ways. The structures that are predictably emergent in these networks react back on behavior in organizations and fields in a process of continuous restructuration that is partly predictable but also involves transformations or transitions that will not appear locally predictable to network actors. The understanding of these dynamical feedbacks and tipping points represents a neglected area of organizational theory.

\subsection{Organizations and Fields}

Formal organizations are enduring structures characterized by legitimate authority relations and mutual rights and obligations among members (Weber, 1968; Leaf, 2004). These structures coordinate production (Chandler, 1977), transactions (Coase, 1937), information flows (March and Simon, 1958; Stinchcombe, 1990), the purposes and consent levels of individual participants (Barnard, 1938; Simon, 1947) and, we contend, the network connections among the people and groups occupying them.

Fields structure inter-organizational relations in an equally distinctive fashion. Institutional fields are supra-organizational transactional linkages that configure the search and regulation systems that govern the interpretation and actions of both organizational and individual participants through formal precepts and monitoring, normative patterns of action, and taken-for-granted cognitive schemas (Powell and DiMaggio, 1991). Several theorists have linked a conception of fields to ties among diverse participants in an activity (Bourdieu, 1992; Sewell, 1992). When placed in the context of the evolution of inter-organizational networks, this conceptualization suggests the reciprocally structuring effects of relational topologies and institutional fields (Giddens, 1986; Powell, 1990). 
We contend that organizations, which formally structure relationships among individuals and collectivities to achieve particular goals, are often characterized by differentiated cohesive groups whose overlaps can draw disparate units and activities closer together. In contrast, fields are commonly organized by more integrative, nested, and searchable topologies where different levels of cohesive embeddings represent both steps on a ladder of integration into the field and windows for search within it.

In the following sections, we develop these propositions in terms of network theories about links between micro-dynamics of attachments in networks and more macro structures of cohesive embeddedness before distinguishing different network topologies of cohesion and offering two propositions about the structural correlates of organizations and fields. Finally, we illustrate our concepts and models with research findings from informal social events, at one level, professional activities (coauthorships) at another and contractual networks in the biotechnology industry at a third level. These collectivities-networks of interacting individuals, groups of individuals, and networks of organizations—-bear distinctive imprints of both fields and organizations.

\subsection{Defining Fields and Organizations as Networks}

To make these propositions more precise, we must first define fields and organizations as networks. A network as a formal construction is a set of nodes and one or more sets of ties. Ties may include sets of edges that correspond to unordered pairs of nodes or directed edges called arcs defined by ordered pairs. We will introduce a formal definition of structural cohesion as a property of network structure (Moody and White, 2003) that is directly measurable and yields powerful predictions about the behavior and dynamics of networks, organizations and fields.

In structural terms, organizational networks are distinguished by internal ties that tend to have more cohesive boundaries as compared to the more extensive and less cohesive overall networks that include their external ties. The cohesive subsets within the organizational boundaries help define differences in their scale, power, and relationship to groups of individuals that occupy and link them. Systems of ideas, values, and normative roles attach to organizations by virtue of relatively stable and cohesive interactions among their members. Individuals, however, will typically belong to multiple organizations and subgroups within them, and to various affinity groups. Thus interpersonal connections may cross organizational boundaries in addition to spanning internal divisions. If it is partly the cohesion of interactive ties within organizations that tends to give a highly orderedeven if fluidly changeable-structure. It must be remembered that organizations interact purposefully within changing environments.

At their simplest, fields are also networks of interactions that may be modeled by arcs, edges and nodes that change over time. Fields are those networks that emerge as structured and structuring environments for organizational and individual participants. To understand organizational dynamics we need to look with equal care to the external ties of organizations within those changing environments. In short, while we treat the network instantiations of organizations and fields as distinctive for the purposes of clarity, a more realistic approach asks how intra and inter-organizational relations 
interact dynamically to co-constitute coordination mechanisms for both organizations and fields.

\section{Theoretical Background}

\subsection{Social Embeddedness}

Embeddedness (Granovetter, 1985, 1992) is a central concept in economic sociology and one of the most commonly cited ideas in the networks literature. It provides a helpful foundation to our work on structural cohesion. Within the context of richly differentiated networks, social, political, economic, and familial relations can provide interlocking configurations of multiplex and cohesive support for one another. Embeddedness, as formulated by Granovetter (1985), is a property of structures in which actors that are integrated in cohesive clusters or multiplex relations of social networks face different sets of opportunities and constraints than those who lack such connections or encumbrances. Granovetter (1992, p. 33) delineates the key division between "local" and "structural" embeddedness:

"Embeddedness" refers to the fact that economic action and outcomes, like all social action and outcomes, are affected by actors' dyadic (pairwise) relations and by the structure of the overall network of relations. As a shorthand, I will refer to these as the relational and the structural aspects of embeddedness. (italics in original.)

He further specifies (p. 35) his understanding of structural embeddedness as the degree to which actors are involved in cohesive groups:

[T]o the extent that a dyad's mutual contacts are connected to one another, there is more efficient information spread about what members of the pair are doing, and thus better ability to shape behavior. Such cohesive groups are better not only at spreading information, but also at generating normative, symbolic, and cultural structures that affect our behavior.

\subsection{From Embeddedness to Cohesion}

Granovetter's description of structural embeddedness suggests that organizational theorists' attention should focus on identifying cohesive subsets in social networks. While the crucial mathematical concept for emergent cohesive subsets in networks was discovered by Menger (1927), a central element missing in most social and natural science network studies has been an adequate theoretical conception and measurement of the concept, which we term structural cohesion (Moody and White, 2003). Structural cohesion has two distinct but deeply equivalent facets. One is cohesion via $k$-node-connectivity: a network is $k$-connected when it is invulnerable to disconnection by removal of fewer than $k$ of its members. "A group's structural cohesion is equal to the minimum number of actors who, if removed from 
the group, would disconnect the group" (Moody and White, 2003, p. 109). More formally (White and Harary, 2001), a maximal set $\mathrm{S}$ with respect to a property $\mathrm{P}$ is one for which every broader set containing $\mathrm{S}$ lacks property $\mathrm{P}$, and a $k$-component is a maximal subgraph of a graph $\mathrm{G}$ that is $k$-connected. When we use the term multiconnectivity we are referring to $k$-connected graphs, their $k$-components, and the fact that all nodes in a $k$-component are $k$-connected.

The other facet that makes membership in $k$-components an appropriate, strong and scalable measure of network cohesion is that Menger proved that a group with structural cohesion $k$ (members of a $k$-component) has at least $k$ node-independent paths between every pair of members, and vice versa. Paths are node-independent if they have only the start and end nodes in common, so that multiconnectivity implies additive strength of redundant ties between pairs of nodes with a cohesiveness proportional to the number of their node-independent paths. Because it specifies a formal identity between a key structural characteristic of graphs and a basic property of network traversal redundancy, graph theorists regard Menger's theorems of $k$-connectivity as one of their half-dozen or so most fundamental discoveries (Harary, 1969; Diestel, 2002, p. 55). We use the term multi-connected for a pair of nodes or a graph whose $k$-connectedness is 2 or more.

The identification of structurally cohesive groups and their nesting in a network is deterministic, with a result that assigns each node to one or more $k$-components (Harary, 1969). The $k$-components of a graph are partially ordered: Any $k$-component with $k>1$ has a unique $(k-1)$-component whose nodes include those of the $k$-component. In addition to $k$-components there are further levels of structural embeddedness wherever clusters are separated within a $k$-component after removal of $k$ of its nodes. Moody and White (2003) provide a tractable algorithm (also implemented in NetMiner, 2004, p. 15) for enumerating cohesive sets, including $k$-components, by levels of embedding.

Whether structural cohesion and embeddedness matter-their consequences and antecedents - has been investigated under the rubric of Predictive Social Cohesion (PSC) theory, which predicts a wide range of consequences. The theoretical and empirical implications of this formalization for social life were examined in Moody and White (2003), who found it to be successful net of other factors in predicting school attachment in high schools and coordinated political action among firms in the Fortune 500. Powell et al. (2004) draw on PSC theory for hypotheses about the formation of contracts in the evolution of the biotech industry. Brudner and White (1997) use PSC theory to predict and validate social class membership at the community level. Structural cohesion should matter to individuals and groups because it scales the linkage of specific actors to one another, facilitates the flow of information and exchange through multiple channels, allows the cross-checking of information as a basis for establishing reliability and trust, and supports the robustness of social groups and their adaptive resilience through multiconnectivity.

\subsection{Co-Evolution of Organizations and Fields as Networks}

2.3.1. The Effect of Micro-Dynamics. How attachments are made in networks (microdynamics; see also Snijders, 2001; Robins et al., in press) structures network topology which in turn alters the opportunity space, constraints and perception of network environments, 
thereby reacts back on micro-behavior and dynamics. Here we put these links in the context of organizations and fields, then examine probability models of micro-dynamics and their various effects on network topology. The reader wishing to avoid technical detail may skip over these probability models, but the regularities summarized here form the basis for the propositions that follow.

To illustrate how network dynamics, the structure of fields and the behavior of actors or organizations co-influence one another, we (1) take field structure as the larger ('macro') environment of organizational and actor ('micro') behavior, (2) focus on network dynamics as the key to their co-evolution, and (3) develop insights from prior research on the cohesive properties of networks. This approach allows us to frame some of the linkages by which micro-macro co-evolution proceeds. Changing patterns of network ties congeal into emergent structures and these emergent forms shape future tie formation in definable ways. Institutionalized groupings may become more stable over time, both at the field, 'macro', level and the organizational or 'micro' level of actors occupying positions in network neighborhoods. As such, a view of organizations and fields as simultaneously emerging from and shaping the networks of relationships reprises Coase's (1937) classic insight that firms and markets offer alternate means to coordinate the complex transactions required for production (see White, 2002). Organizations and fields, in our view, represent complementary mechanisms that structure patterns of relations. Organizations as more purposive, authority driven instrumentalities. Fields are looser, with fewer constraints on interaction, and both give and regulate access to resources and information external to organizations.

2.3.2. Random, Degree-Biased and Mixed Graphs. To conceptualize the link between network dynamics and topology, we begin with three probabilistic models of how successive attachments may be generated in networks with a fixed number $N$ of nodes and a growing number $m$ of edges. All three are two-stage models with a fixed probability $P(u)=1 /(N-1)$ that node $u$ will be one of the two nodes in a new attachment. They differ in the probabilities of $u$ attaching to a second node, $v \neq u$. In equiprobable attachment (Erdös and Rényi, 1961) the attachment probability is constant for all $v$, so that $P_{E}(v)=1 /(N-1)$. These are called random graphs because a tie between any pair of nodes where $u \neq v$ is equiprobable. In degree-biased attachment the probability of an attachment from $u$ to $v$ is proportional to the number of existing links of node $v_{d}$ (of degree $d_{v}$ ), $P_{D}\left(v_{d}\right) \sim d_{v}+\varepsilon$, where $0<\varepsilon \ll 1$ serves to start the process from a graph with no edges. In mixed attachment, $P_{M}(v) \sim$ $d_{v}+A+\varepsilon$, so that $P_{M}(v) \approx P_{D}(v)$ if $A=\varepsilon \ll 1$, and $P_{M}(v) \approx P_{E}(v)$ as $A$ goes to infinity (Dorogovtsev and Mendes, 2003).

2.3.3. Random Graph Topologies. The network topologies of the random graph and the degree-biased graph differ markedly. As the number $m$ of edges in an equiprobable graph grows, the expected topology changes as $m$ goes from 0 to $N / 2$ from a graph with small disconnected subgraphs that grow linearly in number with $m$ to one with a single large component that dominates the others in size (Erdös and Rényi, 1961). The large component grows much more rapidly as $m$ goes from $N / 2$ edges to $N$ edges, following an expected growth curve that is logistic or S-shaped. By the time that $m=N$, almost all random graphs have a giant component that is orders of magnitude larger than every other component. 
As the number $m$ of edges grows from $N$ to $2 N$, a further sigmoid transition occurs for the growth of the largest bicomponent, which almost invariably develops within the giant component, as Moody (2003, p. 38) shows by simulation. As $m$ grows larger, successive $k$-components must form, and as they grow large they are almost always members of the largest $(k-1)$-component. Thus, this nested form of a single cohesive hierarchy is a characteristic outcome even of purely random interaction. This has implications for our argument regarding co-evolution of macro-micro-levels, as between global structural and local behavioral properties of networks.

2.3.4. Mixed Graph Topologies. A mixed attachment process has a variable proportion of random edges. The growth in number of random edges generates a subgraph that follows random graph evolution of $k$-components. For real-world networks, a higher proportion of random ties, averaging over graphs with other attachment processes, translates to a higher probability of a single dominating hierarchy of large $k$-components. This largest cohesive hierarchy of a network with a high proportion of random ties may become a collective, self-organized entity. To the extent that fields have fewer constraints than organizations, and more attachments that are random, their networks will have a greater tendency to form a single cohesive hierarchy among the largest $k$-components and the 'giant' connected component will tend to span the network when the number of edges exceeds the number of nodes. These properties of random graphs provide the motivation for defining a network topology of 'mono-cones' (Section 3.1) that contrasts with what is expected in the case of degree-biased graphs. In the mixed attachment probability model, networks are generated where if $A \approx 0$ the expected exponent $\alpha$ of the power-law degree distribution approaches 3 asymptotically from below as $N$ goes to infinity (Barabási, 2003; proven by Bollobás and Riordan, 2003) but as $A>0$ grows larger, $\alpha$ decreases.

2.3.5. Degree-Biased Graph Topologies. Barabási (2002, 2003) popularized preferential attachment to degree as a dynamic model for large networks. A degree distribution is said to follow a power law if the probability of having $k$ neighbors is approximated by $p(k) \sim k^{-\alpha}$. The exponent $\alpha$ is estimated as the linear slope in a scatterplot with log $k$ (number of neighbors) on the $x$ axis and $\log N_{k}$ (number of nodes with $k$ neighbors) on the $y$ axis. Degree-biased graphs generate histograms of degree frequency that follow a power-law, where the 'fat tails' of the distribution correspond to network 'hubs' with degree frequencies orders of magnitude larger than expected in a random graph with the same mean degree. In degree-biased graphs the common sets of nodes attached to multiple hubs tend to form larger multiple overlapping cohesive hierarchies. Only when the power-law exponent is in the range 2-2.3 do the hubs tend to connect to form a single cohesive hierarchy (Adamic et al., 2003). White and Johansen (2004) found that the degree-biased graphs with powerlaw exponents between 2-3 listed by Barabási (2003) tend to have exponents in the 2-2.3 range once those with broken-scale slopes are removed (e.g., different exponents for English word co-occurrences for high- vs. low- frequency words). This may indicate selective biases in the real-world evolution of networks. These networks, within the 2-2.3 range, are also orders of magnitude larger on average than those with exponents between 1 and 2, which are expected to have 'multi-cone' typologies (Section 2.4). 
2.3.6. Locally-Biased Attachments. Mixed probability models may also include local attachment biases (Rapoport, 1957) such as reciprocity, transitivity, or co-parent/co-child biases (two nodes with directed links to the same other having a tendency to attach). Evidence of such biases can be derived from a triad census (Batagelj and Mrvar, 2001; Davis and Leinhardt, 1972). Locally-biased attachments are ever-present within organizational networks, and mixes involving pervasive locally-biased attachments along with random and degree biases commonly inflect degree distributions away from power-law distributions or lower the exponent, thereby altering the global network topology.

2.3.7. Assortative Mixing Attachments. Another potential micro-macro linkage for network structure derives from the extent to which degree values correlate over edges, i.e., assortative or disassortative mixing by degree. Characteristic negative or positive degree correlations, according to Newman and Park (2003), are one of the more significant distinctions between social and nonsocial networks. They contrast assortative mixing in social networks, enabling community formation, with the disassortative mixing common to technological and biological networks, where high-degree nodes have a higher probability of attaching to nodes of low degree (Maslov et al., 2002; Amaral et al., 2000). In assortative mixing, in contrast, nodes with higher degree have higher probability of connecting to other nodes of higher degree, controlling for expected probabilities given the nodal degree distribution. The topological inflection expected from positive degree correlations (assortative mixing) is towards a single large 'mono-cone' cohesive hierarchy.

2.3.8. Arbitrary Degree-Distribution Biases and the Study of Searchability. Newman's et al. (2001) generating function method allows the simulation and measurement of the size and distribution of $k$-components for a random graph with the same degree distribution as an empirical graph, and provides Monte-Carlo models for "expected topologies" of networks with the observed degree distribution. Adamic et al. (2003) used this method to discover that among networks with power-law degree distributions, only those with exponents in the range 2 to 2.3 had a cohesive topology that allows searchability: the capacity to move through successive hubs in connected local neighborhoods to find an arbitrary target in orders of magnitude fewer moves than required in a random search (Kleinberg, 2000).

\section{Definitions for Types of Cohesive Topologies}

\subsection{Definitions, 1}

Recall that a $k$-component is maximal if no superset has connectivity $k$. The breadth of a $k$-component (see Section 2.2) is $|N k|$, the number of nodes in its node set $N k$, and its depth is indexed by its cohesion contour level $k$. A cohesive cone of a graph $G$ is a maximal set of nested $k$-components of $G$ (i.e., in which every $K_{1}, K_{2}$ pair of distinct $k$-components for which $K_{1}$ is of equal or lesser depth than $K_{2}$ is ordered by set inclusion of its node sets, $N K_{1} \subseteq N K_{2}$ ). The depth $h$ of a cone is the largest contour number of its $k$-components and is uniquely defined because they are fully ordered from 1 to $h$. A mono-cone of $G$ is a cone with no nodes in common with other cones in $G$. No cone can have a single 
node at its greatest connectivity $h$. There are always 3 or more cyclically-connected nodes at this connectivity since cones by definition have a cohesive subset of connectivity 2 or more.

Cones thus lend themselves to thinking of cohesion as a collective phenomenon, not a matter of inequalities between individual nodes. Further, network hubs with a degree $k$ that is higher than their greatest contour $c$ in a cone will have only the cohesion $c<k$ of their highest cohesive contour.

The 'depth' of connectivity for actors in a cohesive cone nicely captures the intuitive sense of being involved in relations that are structurally embedded in a social network, in direct contrast to "arms-length" connections (Uzzi, 1996). As such, one aspect of structural embeddedness - the depth of involvement in a cohesive structure-is captured by the nesting of individuals, groups, or organizations in $k$-components.

Nested cohesive hierarchies, as in Section 2.3.3, offer one possible outcome of network evolution. A second type of outcome, one of dispersion and overlap among cohesive clusters, might co-evolve with non-equiprobable attachments of actors or organizations, as in degreebiased or mixed-bias graphs (Sections 2.3.4-2.3.5). The contour level of a node is the largest $k$ for a $k$-component of which it is a member.

Theorems in graphical evolution commonly deduce findings about concepts that are looser than most mathematical definitions, such as deriving properties of 'almost all graphs.' In this vein, we define a graph $G$ with a mono-cone (macro) topology as one in which the mono-cone of greatest depth has $k$-components that are orders of magnitude broader than those of similar depth $k$ for other mono-cones in $G$.

\subsection{Definitions, 2}

A multi-cone of a graph $G$ is a set of cones of $G$, each having nodes in common with others in the set. $G$ has a multi-cone (macro) topology if its largest multi-cone has multiple intersecting cones with $k$-components at similar depths and with similar orders of magnitude in breadth, and if its $k$-components are deeper or, if of similar depth, orders of magnitude broader than those of the mono-cones in $G$.

Analysis in terms of multi-cones differs from many density-driven approaches to cohesion and clustering. The minimal density for a $k$-component with $n$ nodes is $k /(n-1)$, so it is possible to have various types of "structural holes" (Burt, 1992) within $k$-components and within circles of intersecting $k$-components. Such circles, however, cannot involve $k-1$ nodes in each intersection because the ensemble would then be a single $k$-component rather than a set of distinct $k$-components. This constraint can produce some interesting asymmetries in organizational networks.

The definition of a multi-cone topology allows multiple disconnected multi-cones with diverse $k$-component properties. Thus, a third type of outcome of network evolution is one of non-overlap among multiple cohesive clusters. Because discrete mono-cones are at most 1-connected, we do not discuss them here because they comprise a non-cohesive network topology, and such networks lack the field or organizational properties we associate with cohesion. 


\section{Case Studies}

We have chosen three examples to provide a contrast set that includes: (1) large and small networks; (2) 1- and 2-mode networks; (3) exact and approximate calculation of cohesive embeddings; (4) different mixes of organizational and field properties and of organizations within fields; (5) examples of the contrast between $k$-components and other cohesively embedded subsets; and (6) greater and lesser tendencies towards degree-bias or randomness in attachments. We first discuss each case in some detail and then present a proposition relating to our broader interest in the cohesive characters of organizations and fields and to the properties of the example.

\subsection{Case 1: Cohesive Hierarchy in Davis's Southern Women Events Network}

We examine data collected on women's attendance at society-page events in a nine month period during the 1930s. The data derive from a sociological study conducted in a town in the deep south. This network illustrates a number of points about cohesion. First, although a similar analysis could be done of social relationships among individuals (a 1-mode network), this example shows how cohesion analysis is equally appropriate to a bipartite (2-mode) network of actors. The concept of cohesion is applicable here to the relationships among the women, among the events, and within the ensemble of women-and-events. Second, it is a network with a temporal dynamic since the events are ordered in time. Third, it illustrates a mono-cone topology, and how any $k$-component with $k>1$ is necessarily also cohesive at level $k-1$. Fourth, it displays the difference between $k$-components proper and other embedded subsets within the deepest $k$-component of a cone. Fifth, since these data have been analyzed repeatedly, our results may be compared with studies that use different tools for network analysis. For this comparison, the reader should consult Freeman's (2003) meta-analysis of all previous studies of the Southern women/events network.

Figure 1 derives from the attendance matrix recorded by Davis et al. (1941, p. 148). Women are shown as darker nodes with first names, and events as lighter nodes with numbers in their interior. The larger-sized numbers attached to events, 1 to 14 , give the temporal sequence. The scaling of nodes is a spring-embedding that pulls nodes together when they are attached and pushes them apart when they are not attached. Spring-embedding is consistent with the idea of scaling-by-cohesion, whereas a spatial scaling such as correspondence analysis puts nodes together when they have the same attachments even if they are not connected.

The concentric solid-line contours around sets of nodes are labeled for the successive $k$-components of the graph: Fourteen women and nine events are in the 4-component; fifteen women and thirteen events in the 3-component; and the network as a whole is biconnected (a bicomponent). The women who share membership in more cohesive $k$-components of the mono-cone represent the more embedded structural collectives. Thus relations among Helen, Laura, and Theresa in figure 1, for instance, have more deeply embedded connections with one another than with Olivia, Flora or Dorothy.

The tree-like structure to the right of figure 1 displays the results of the Moody-White algorithm (except for cuts that separate single nodes) as it searches for every $k$-component 


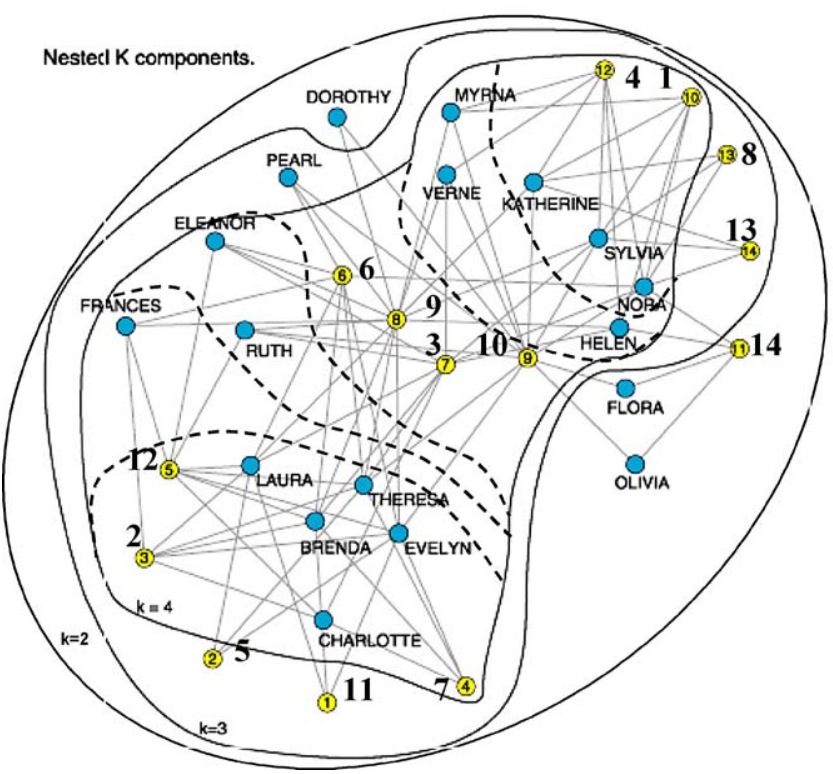

Moody-White (2003) algorithm K-Cut History

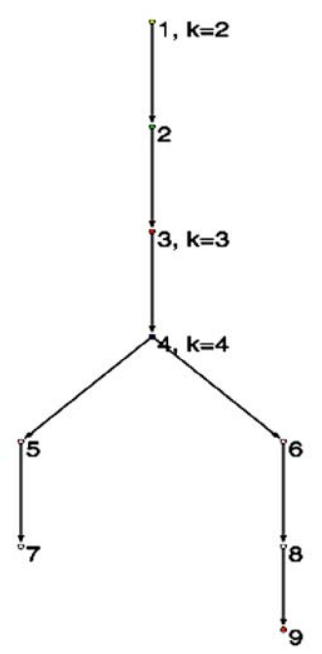

Figure 1. A hierarchy of nested $k$-components (4-cone), with cohesive contours. Key. The $k$-components identified by the Moody-White (2003) algorithm within the bipartite graph of events and actors in events are enclosed in solid-line cohesive contours and labeled $k=2,3$ and 4 . The larger numbers 1-14 give the temporal order of events. The branching tree at right shows the order in which the algorithm finds first the $k$-components (again labeled $k=2,3$ and 4) and then the remaining embedded subsets that remain following removal of four central events within the 4-component. The dotted lines in the graph separate the 1- and 2-components identified at steps 5 and 7 by the algorithm, and the 1-, 2-, and 3-components identified at steps 6, 8 and 9. These sets are separated out within the 4-component by the dashed lines for subsets on opposite sides of the 4-component split. The only other four node 4-component split (also found by the algorithm but not shown) places temporal event 6 in the lower-left embedded set and removes Nora from the upper-right embedded set.

and structural embedding in the graph. It identifies the successively more cohesive $k$ components labeled $k=2$ through $k=4$. The 1- and 2-components completely overlap and contain nested three and four components. As graph fragments are pared away to locate these structures, once the most cohesive component is identified, the algorithm proceeds to split this 4-component into the only two subsets that can be separated by removal of four events (cutsets of size 4, or 4-cuts), and then to analyze cohesion when each of these two subsets is considered on their own. These further levels of structural embeddedness are demarcated in figure 1 by the concentric regions enclosed by dashed lines within the 4-component.

Cohesive embeddings within $k$-components are not unique when found by removal of $k$ nodes, which is the last step in the Moody-White algorithm. Finding multiple overlapping sets, however, can again be a useful result. There are two such 4-cuts within the Southern women 4-component: One removes four central events to separate two opposing 
clusters of structurally embedded women and events, and the other removes three of these central events plus one woman to give a slightly different result as to the placement of this woman (Nora) in defining boundaries between the opposing clusters. These divisions agree in the main with the findings of other methods, but also display a basis for the variability that we find in comparing their results (Freeman, 2003). The 4cuts for the broadest structurally embedded clusters divide into three sets: (A) events 1-5 (or 1-6) (occurring in temporal order 2, 5, 7, 11 and 12) and the women who attended them; (B) events 10-14 (occurring in order $4,8,13,14$ ) and the women who attended them (C) the cut-set of events 6-9 (or 7-9) that include some women from the both clusters.

By keeping the bipartite network intact, the structural cohesion approach is able to simultaneously model the full ordering of $k$-components, the partial ordering of cohesively embedded sets, and the temporal ordering of events. Event dynamics may be viewed at http://eclectic.ss.uci.edu/ drwhite/dynamics/DavisTime002.htm for a series of 14 slides, one for each event in the time-series. Viewed dynamically, the transitions among events move between sets (C-A-B-C-A-B-A-C-B-A) for the first nine transitions but not the last four (B-B-A-A-C-C; $p=.03$ ), showing a dynamic with a mix of randomness and repetitive sequencing that alternates between competitive and integrative events.

\subsection{Implications of Mono-Cone Topology (Proposition 1)}

Networks as Fields. Based on the probability models in Section 2.3, we expect networks to be more likely to represent fields when they have a mono-cone cohesive topology or single stacked multiconnectivity hierarchy. Some of the processes that contribute to monocone cohesive topologies are random attachments (Section 2.3.2), locally-biased attachments (Section 2.3.6), degree-biased (Section 2.3.5) and mixed (Section 2.3.4) attachments with power-law exponents on degree distributions between 2-2.3, and assortative mixing (Section 2.3.7). Our case 3 (Section 4.5) will exemplify the last two properties plus attachment bias to cohesion. Degree-biased and mixed attachments with exponents less than 2 (Sections 2.3.4-2.3.5) will tend to produce multi-cone topologies.

The Southern women network in figure 1 illustrates the nested structure of cohesion in a mono-cone topology. While small, the Southern women's network is a good example of a field. There is organization here, but it is informal and emergent, with clustered sets of women organizing different events and inviting members of their clusters or larger community, and some invitees attending and some not at each level. Within each of the most deeply embedded sets of women who associated in opposing event-sets, individual records of attendance are highly variable: Freeman and White (1993) show with a lattice representation the near-random intersections of attendees within each cluster. Still, the latent organizational tendencies in the oppositional structural embeddings within the 4-component indicate informal organization into subgroups (somewhat like multi-cones having a common 4-cohesive contour). The timing of which of these two latent groups sponsor which of the events, however, is irregular, even if turn-taking or competition is involved in the dynamics. 


\subsection{Case 2: Visualizing Multi-Cone Topologies in a Large Network}

We move next to the case of a social science co-authorship network, with a particular focus on the largest bicomponent ( $N=29,462$ authors) of papers listed in Sociological Abstracts from 1963-1999. Because computation of $k$-components in large networks is prohibitively time consuming, we map cohesive contours using an approximation technique developed by Moody (2004). His results in figure 2 show a multi-cone structure.

Figure 2 shows the $3 \mathrm{~d}$ and $2 \mathrm{~d}$ results of Moody's procedure for large networks that finds approximate cohesive contour plots instead of computing $k$-components directly. $\mathrm{He}$ built on the fact that a force-directed graph or spring-embedding algorithm will draw nodes near one another with force proportional to their cohesion and push them apart the lower their cohesion. He calculated the number of nodes within $x y$ squares of constant areas in the spring-embedder scaling of the network and then plotted a smooth probability density function (bivariate kernal density) over the squares in the $x y$ surface. This approximation procedure maps relative contours, some of which wrap around multiple cohesive cones (shown as peaks in figure 2) and allows estimation of a structural embedding variable that varies by node according to the density of the $x y$ square where it is located. Two clear peaks appear in the relative contour plot, one significantly taller than the other. The major peak corresponds to people writing in general sociology, while the shorter peak corresponds to people writing largely on topics related to public health. The $2 \mathrm{~d}$ contours show two distinct cones above contour 2.74 (one with two subcones with slight rises in contour at the depression) and a contour at 2.11 in the $3 \mathrm{~d}$ image that wraps around both the larger peaks as well as the lower cones.

Organizational networks need not be small in size, and co-authorship networks, by virtue of the distinctions imposed by the formal architecture of sub-disciplines, focused specialty journals and training programs, may be taken to represent structural mid-points between organizational networks and more open fields that tend toward mono-cone cohesion. The plots for this network show a multi-cone topology, consistent with the probability models that we expect to apply to organizational networks (Sections 2.3.6 and 4.4, below).

\subsection{Implications of Multi-Cone Topology (Proposition 2)}

Organizational Networks. Organizations tend to impose boundaries between differentiated subgroups in their division of labor. Within organizations we expect multiple overlapping cohesive groups (and thus multi-cones topologies) that result from the segmentation and networking of individuals and groups under the constraints of interdependent activities and formal lines of supervisory authority and regulation. This proposition corresponds to the idea that organizations have substructures and an internal division of labor. We also expect the observed multi-cones to overlap and cross-cut the formal organizational job descriptions. Of interest is how overlapping cohesive cones integrate the organization, and what are the implications of the overlaps and their sizes compared to those of the intersecting $k$-components.

In terms of micro-macro linkage, the probability models that generate multi-cone structure are locally-biased attachments (Section 2.3.6) and degree-biased and mixed attachments and 

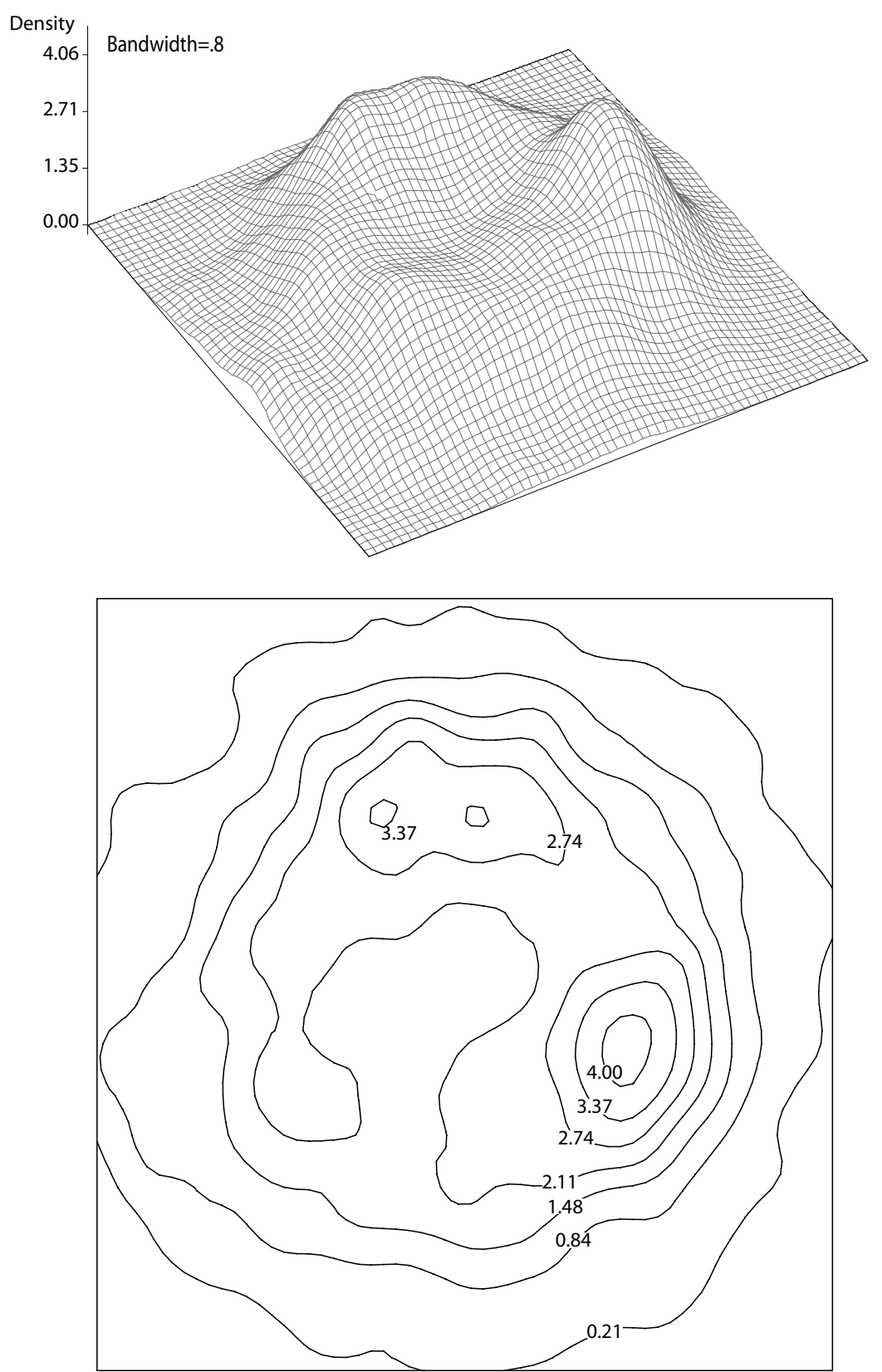

Contour Sociogram. Contour values denote bivariate density estimates, indicating every 15th percent of the range between the 5th and 95th percentiles. $X$ and $Y$ positions represent node placements resulting from Fruchterman-Reingold optimization

Figure 2. Social science coauthorship network largest bicomponent $(n=29,462)$. 
topologies (Sections 2.3.5 and 2.3.4) in cases where the power-law degree distributions have exponents less than 2 . Where the exponent exceeds 3 virtually no cohesion is expected in the graph, so the expected topology is neither mono-cone nor multi-cone.

Multi-cone topology focuses on overlaps among cohesive $k$-components and embeddings. Topological variants in this class capture many of the properties associated with ideas about social spaces or manifolds that form the communicative spines of organizations (Friedkin, 1998). What defines a multi-cone structure is a maximal subgraph of $k$-components in which some of the contours around the $k$-components embed the contours of multiple cones. These specific multi-cone contours hold together various disjoint structures of higher multiconnectivity. Multi-cone contours ('ridges') are thus a potentially powerful and predictive feature of organizations. When some of the $k$-components in these ridge-type structures overlap with only a single node in common, a situation may arise that Likert (1961) calls organizational link-pins: identified sets of individuals who belong uniquely to two distinct cohesive groups (e.g., where they might be a supervisor in one and supervised in another). Similarly, Likert's model of an organizational short-circuit is a cohesive group that spans three levels of supervisory authority (and overlaps with other cohesive groups) and can be modeled by a combination of cohesive groups and supervisory relations.

\subsection{Case 3: Biotechnology Collaborations as a Field of Embedded Networks}

Powell et al. (2004) analyzed the development and elaboration of the field of biotechnology, showing how the formation, dissolution, and rewiring of new and repeated network ties over a twelve-year period, from 1988 to 1999, shaped and reshaped the opportunity structure of the field. For this network, the idea of a field captures the diversity of organizations more aptly than any other term, such as industry or population. Universities, government labs, and nonprofit hospitals and research institutes are a critical part of the field; while on the commercial side, established pharmaceutical firms and dedicated biotechnology companies are involved in bringing new medicines to market. In this commercial field of the life sciences, cohesive blocks play a major role in the network dynamics. We also observed degree-biased power-law distributions (Section 2.3.5) for firms in biotechnology, which are typical of networks with preferential attachments to degree and a tendency towards the formation of central hubs. Deep empirical knowledge of the industry, however, led us to question whether this power-law tendency was not due to a degree-based attachment bias toward more central nodes (Section 2.3.2) but to a preference for access to well-connected but diversely affiliated nodes that had the ability to recognize and access novel information, and hence a cohesion-biased attachment process.

Hence, we investigated the hypothesis of preferential attachment to structural cohesion in the biotech industry. A series of network visualizations highlighted both the evolving topology of the field and the processes by which new ties and organizations were added. We turned to a statistical examination of network formation and dissolution, and assessed the effects of alternative mechanisms of attachment, including rich-get-richer, homophily, follow-the trend, and preference for diversity. One result of the analysis was to show that the processes of attachment changed as organizations aged and their portfolios of connections 
changed. Micro-level choices and macro-network trends were seen to co-evolve in ways that led to shifts in the dominant logics of affiliation.

Rather than a static structure of hierarchical cohesion, this science-based field has a preference for novelty. The most central firms search for new sources of innovation on the periphery and pull the new entrants into the center. This is an example of the search capability referenced earlier (Section 2.3.8 (Adamic et al., 2003): indeed, the exponent for the power-law degree distributions for each of the biotech subsectors is within the 2-2.3 range as expected). The most cohesive biotech cluster appears to have the widest search horizons, while lesser cohesion entails narrower, less multiconnected perspectives. The 'ladder' structure of the mono-cone field represents both an open elite (Owen-Smith et al., 2004), whose members 'prospect' for likely newcomers throughout the network, and a hierarchy that shapes attachment preferences and search behavior across the field.

Figure 3, using Moody's density function method, captures in the scaling contour the mix of a static element (a cohesive hierarchy) and a dynamic element, that of central firms reaching out to the perimeter of the network, which shows up in the scaling contour as a wave effect: a scaling displacement seen in the raised cohesive contour of the outer ring around the central cone in the figure. This secondary structure is an emergent feature of the field created by the Schumpeterian dynamic of the innovation 'pump' in this industry, in which highly cohesive firms reach out to form new ties with those on the cohesive periphery. Drawn after our Powell et al. (2004) analyses were complete, figure 3 helps to confirm the operation and structure of this pump independently of the earlier evidence: Namely, that a core feature of the innovation process is operating through the field of external contracts among firms and the dynamics of new-tie formation.

Figure 3, however, largely fits the structure of a mono-cone, consistent with Proposition 1 in which we argued that fields should have a mono-cone structure. Part of the reason for this prediction is that fields are by definition freer of organizational constraints than organizations per se, so that, as with networks in which ties form randomly (Section 2.3.3), cohesion at the level of the broader $k$-components tends to cumulate into a single hierarchy. In the biotech case, however, there is also strong bias, statistically demonstrable, of attachment to structural cohesion. We might find cases, however, where organizational features of networks characterized by multi-cones and ridge-type structures are emergent within a field at the level of both local neighborhoods (regularities in clusters of interactions) and the overall network.

\subsection{Implications for Multi-Cone Topologies (Proposition 3)}

Multi-Organizational Fields and Difference in Scale. If an organization is more likely to have a multi-cone topology and a field a mono-cone topology, how do we conceptualize a multi-organizational field with an overall mono-cone topology when contained within it are narrower regions of multi-cone organizational networks? This is possible because differences of scale between organizations and fields operate at two different levels. This is reflected in our 'looser' definitions of topological structures, which permit the interlock of contrasting levels in cohesive scale (depth vs. breadth, which relate inversely in a single 

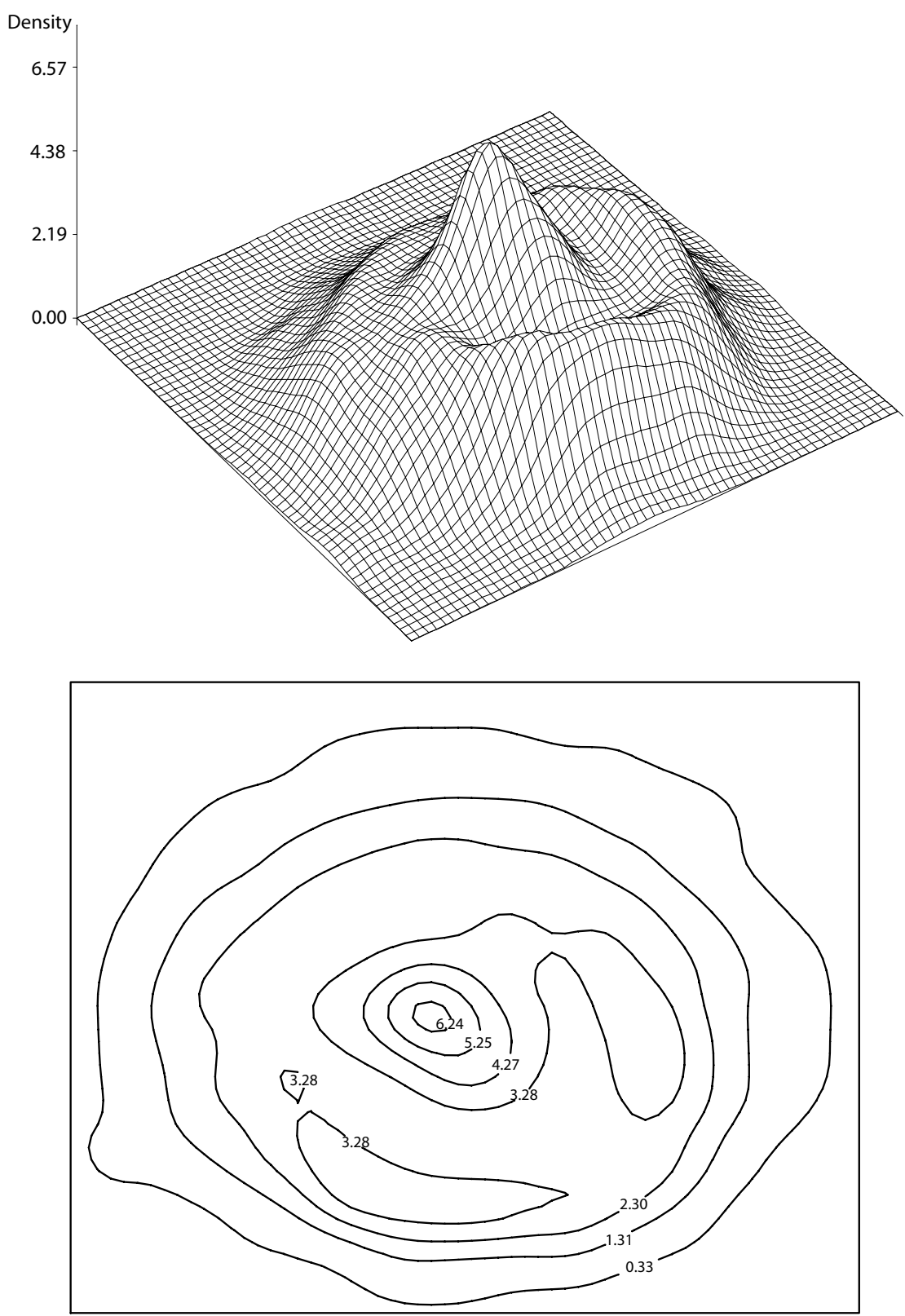

Contour Sociogram. Contour values denote bivariate density estimates, indicating every 15 th percent of the range between the 5 th and 95 th percentiles. $X$ and $Y$ positions represent node placements resulting from Fruchterman-Reingold optimization

Figure 3. Density estimates for the field of biotechnology. 
cone) according to their relative frequencies. The cohesion of an organization is often greater but more narrowly distributed than that of fields (Section 1.2), while that of fields may be shallower but broader in the number of nodes comprised in each of the smaller organizational networks. To be mono-cone in the presence of such smaller organizational structures within its network, the field must have broader $k$-components (i.e., with significantly more nodes) than comparable $k$-components in the intersecting cohesive cones of organizations. The cohesive cones within organizations, then, will overlap with those of the mono-cone field to varying degrees.

In the case of degree-biased network topologies (Section 2.3.5), the presence of many smaller organizational structures within the network will tend to reduce the exponent of the degree distribution to within the range 1-2 and to reduce the likelihood that the network will qualify as a field. We conjecture that when networks with degree-biased network topologies have an exponent between 2 and 2.3, roughly consistent with the presence of cohesion among hubs of the networks, the network will qualify as a field. ${ }^{1}$

\section{Discussion and Conclusion}

Structural cohesion measures, specifically $k$-components and the cohesive cones in which $k$-components are often embedded, provide precise ways to operationalize and test the extent to which hierarchically nested or overlapping subnetworks characterize a given social field or organization within its larger network environment, and to test hypotheses about the consequences of cohesive memberships, overlaps, and network topologies. These concepts, when cast in terms of network topologies, help to link broad organizational and network theories and propositions with specific structural measures and testable hypotheses such as those of Predictive Structural Cohesion theory. At the core of PSC theory is, first of all, an account of how units with greater cohesion act as transmission amplifiers, as organizations utilize the redundancies of multiple channels to overcome the distance decay that typically occurs with single-path network transmission. Second, multi-cone structures help to focus attention on the interfaces between more cohesive subgroups whose occupants may play crucial roles in organizational connectivity, e.g., with respect to supervisory relations, overall communication quality, and coordination. Third, they explain how reliable transmission at a distance can occur between distant social positions in an organization or a field, via higher levels of multiconnectivity operating through intersecting cohesive groups. Fourth, they explain how weak or even strong ties can occur between nodes or individuals that are quite distant, when there is high solidarity in terms of perceived agreement and acknowledged influence. Multi-cone and ridge structures provide a framework for accounts of communicative spines of organizations that are heavily dependent on intermediaries, subgroups, and role positions in complex interlocking positions, and differently configured than mono-cone cohesive structures.

We argued for extending PSC theory to identify the network footprints of fields and organizations as distinct but interlocking concepts, and to study how organizations may gain the benefits of integration into larger fields through overlaps and 'contours' of cohesion. Our goal here was to develop additional analytic vocabulary for the study of cohesive topologies in networks of fields and organizations and to demonstrate the utility of these 
concepts with examples from network studies. Our contrasting definitions of fields and organizations take into account that real world empirical networks mix elements from both levels of observation, just as different probabilistic biases are found in mixed rather than pure form (Section 2.3), and cones with different breadth and depth measures of cohesion may be found in different mixes. Our arguments and explanations are oriented towards identifying a class of strong micro-macro linkages between the behavior of agents in a network context and the network topology that their behavior generates (there are of course many other micro-macro links not discussed here where network processes generate one form of topology or another, see for example: Johnsen, 1985; Snijders, 2001; Robins et al., in press, White and Johansen, 2004, introduction).

Behavior re-tunes in turn to exploit or explore alterations in network structure and perceived dynamics, and the micro-macro feedback process is recursive.

In introducing the concept of field, we observed that fields are conceived as having fewer constraints on interactions than organizations, and we argued from probabilistic models to explain our prediction of the tendency for broad mono-cones to occur in fields as a product of interactions that occur at random and are less structured purely local attachment bias or by degree or 'popularity' biases that fall within certain ranges defined by the exponent associated with such biases (Sections 2.3.4 and 2.3.8). When organizations build heavy degree-biases into their interactions, the micro-macro linkages of probability models (Section 2.3.5) map these biases into greater likelihoods of multi-cone network topologies, for example, when the exponent of the power-law degree distribution is between 2.3 and 3 . This is because these degree distributions entail the creation of cohesive subsets that emerge out of overlaps of sizeable sets of nodes that have different sets of hubs in common but the hubs tend to be disconnected among themselves (Adamic et al., 2003). In contrast, hubs tend to be connected and contribute to formation of a mono-cone field topology when such exponents scale between 2 and 2.3, but when they fall below 2 the topology that is generated tends once again to be multi-cone because of cohesive clusters dominated by local rather than global hubs. While recognizing the importance of power-law distributions by degree (Barabási, 2003), however, many other attachment mechanisms may drive network evolution.

Using the properties of cohesive network topologies, as in our examples, we are better able to learn how to predict social phenomena such as behavior in and evolution of organizations, tie formation in networks, and the dynamics of fields. Our micro-macro propositions one (Section 4.2) and two (Section 4.4), backed by probabilistic models, predict connections between organizational processes structured by formal goals and/or designs and multi-cone network topologies, while field-level processes that are less constrained benefit from monocone network topologies that emerge from more fluid interactions. These propositions offer the beginnings of a more general theoretical base (with transferable measurability of cohesive structures) for understanding how micro-behavior and local network topology connect to the macro-topology of networks, and for understanding the dynamics and feedback loops between the two. Proposition three (Section 4.6) shows how mono- and multi-cone topologies may interlock as fields and organizations through differences in two complementary types of scale, one in breadth of membership of a cohesive set and the other in depth of cohesive embedding. Within a single cone these vary inversely, and deep but narrow (and 
segmented) organizational cohesion is compatible with the broader but shallower cohesion of a field.

A theory of multi-cone structures in organizations fits into the general class of neighborhood-based network processes that are applicable to the study of markets, competition, and organizational fields and communities whose global structure emerges from local processes. When amplified by cohesion arguments, multi-cone structures provide a powerful model of information flows and social influence in organizations and fields be they informal or formal. Friedkin's (1998) work establishes that multiconnectivity has major effects, for example, on interpersonal influence.

Mono-cone field processes appear simpler by comparison to those of multi-cone structures, but when studied dynamically, as in our biotechnology example, they also illustrate complex internal processes. The mono-cone structure of biotechnology is one where flexible and shifting ties span the field and create the type of single hierarchically-embedded cohesive structure that is documented in our examples in figures 1 and 3. Cohesion acts in this field as a radar screen for searching for prospective partners, a ladder for successive attachments, and a source of collaborative resources.

In sum, cohesive hierarchies of different sorts have especially useful and predictive topologies. We have defined and illustrated the two basic forms of cohesive topologies, mono-cone hierarchies and multi-cone ridge structures. By identifying the organizational and field aspects of networks and their micro-macro linkages, we may be in a better position to develop a network basis for organizational theory that is sensitive not only to the internal networks of organizations but also to variations in the type and intensity of network linkages into the fields in which they are embedded.

\section{Acknowledgments}

We thank Alessandro Lomi, Pip Pattison and three anonymous reviewers for comments on earlier drafts of this paper and to Lomi and Pattison as editors for detailed suggestions for revision. Three of us also benefited from preliminary discussions of these issues at the Santa Fe Institute. Direct all correspondence to Douglas R.White, drwhite@uci.edu.

\section{Note}

1. One can speculate that when one organization populates the highest $k$-component of a field the shape of the field is likely to change into that of a multi-cone as the dominant organization acts to reshuffle activities in the field and to restructure cohesive ties to form interlocked and supervised subgroups. Case 2 might represent outcome of this process if we consider the American Sociological Association as the organization that governs the division of publications among journals. Attachment biases to cohesion per se, such as we find in the biotech industry, may also prevent organizational monopolies over the cohesive core of an entire field. Because in the biotech study organizations (and their internal networks) were collapsed into single nodes the problem addressed in proposition 3 did not arise directly but resurfaced as we observed new organizational forms emerge out of inter-organizational relations, as corporations merged and split, bought one another out, and formed long-term strategic alliances. 


\section{References}

Adamic, L.A., R.M. Lukose and B.A. Huberman (2003), "Local Search in Unstructured Networks," in S. Bornholdt and H.G. Schuster (Eds.), Handbook of Graphs and Networks: From the Genome to the Internet. Berlin: WileyEurope, http://arxiv.org/abs/cond-mat/0204181.

Amaral, L.A.N., A. Scala, M. Berthélémy and H.E. Stanley (2000), "Classes of Small-World Networks," Proc. Natl. Acad. Sci. USA, 97, 11149-52, http://polymer.bu.edu/ amaral/Papers/pnas00a.pdf.

Barabási, A.-L. (2002), Linked: The New Science of Networks. Cambridge, MA: Perseus Publishing.

Barabási, A.-L. (2003), "Emergence of Scaling in Complex Networks," in S. Bornholdt and H.G. Schuster (Eds.), Handbook of Graphs and Networks: From the Genome to the Internet. Berlin: Wiley-VCH.

Batagelj, V. and A. Mrvar (2001), "A Subquadratic Triad Census Algorithm for Large Sparse Networks with Small Maximum Degree,” Social Networks, 23, 237-243.

Bollobás, B. and O.M. Riordan (2003), "Mathematical Results on Scale-Free Random Graphs," in S. Bornholdt and H.G. Schuster (Eds.). Handbook of Graphs and Networks: From the Genome to the Internet. Berlin: Wiley-VCH, pp. 1-33.

Bourdieu, P. (1992), "The Logic of Fields," in P. Bourdieu and L. Wacquant (Eds.). An Invitation to Reflexive Sociology. Chicago: University of Chicago Press, pp. 94-114.

Brudner, L.A. and D.R. White (1997), "Class, Property and Structural Endogamy: Visualizing Networked Histories," Theory and Society, 25, 161-208, http://eclectic.ss.uci.edu/ drwhite/ T\&S/BrudnerWhite1997Convert.pdf.

Burt, Ronald S. (1992), Structural Holes: The Social Structure of Competition. Harvard University Press.

Coase, R. (1937), "The Nature of the Firm," Economica, 4(16), 386-405, New Series, http://www.jstor.org/ view/00130427/di009864/00p00034/0.

Davis, A., B.B. Gardner and M.R. Gardner (1941), Deep South. Chicago: The University of Chicago Press.

Davis, J.A. and S. Leinhardt (1972), "The Structure of Positive Interpersonal Relations in Small Groups," in J. Berger (Ed.), Sociological Theories in Progress. Boston: Houghton Mifflin, vol. 2, pp. 218-251.

Diestel, R. (2002, electronic edition), Graph Theory. New York, NY: Springer-Verlag, http://www.math.unihamburg.de/home/diestel/books/graph.theory/GraphTheoryII.pdf.

DiMaggio, P.J. and W.W. Powell (1983), “The Iron Cage Revisited: Institutional Isomorphism and Collective Rationality in Organizational Fields," American Sociological Review, 48, 147-160.

Dorogovtsev, S.N. and J.F.F. Mendes (2003), Evolution of Networks: From Biological Nets to the Internet and $W W W$. Oxford: Oxford University Press.

Erdös, P. and A. Rényi (1961), "On the Evolution of Random Graphs,” Bulletin of the Institute of International Statistics, 38, 343-347.

Freeman, L.C. (2003), "Finding Groups: A Meta-Analysis of the Southern Women Data," in R. Breiger, K. Carley and P. Pattison (Eds.). Dynamic Social Network Modeling and Analysis. Washington: The National Academies Press.

Freeman, L.C. and D.R. White (1993), "Using Galois Lattices to Represent Network Data," Sociological Methodology, 23, 127-146. Web site: http://www.jstor.org/view/00811750/ap010023/01a00050/0.

Friedkin, N.E. (1998), A Structural Theory of Social Influence. New York: Cambridge University Press.

Giddens, A. (1986), The Constitution of Society. Berkeley: University of California Press.

Granovetter, M. (1985), "Economic Action and Social Structure: The Problem of Embeddedness," American Journal of Sociology, 91(3), 481-510.

Granovetter, M. (1992), "Problems of Explanation in Economic Sociology," in N. Nohria and R. Eccles (Eds.). Networks and Organizations. Boston, MA: Harvard Business School Press, pp. 25-56.

Harary, F. (1969), Graph Theory. Addison-Wesley Publishing Company.

Johnsen, E.C. (1985), "Network Macrostructure Models for the Davis-Leinhardt Set of Empirical Sociomatrices," Social Networks, 7, 203-224.

Kleinberg, J. (2000), "Navigation in a Small World," Nature, 506, 845, http://www.cs.cornell.edu/ home/kleinber/nat00.pdf.

Leaf, M. (2004), "Cultural Systems and Organizational Processes,” Cybernetics and Systems, 10, $289-313$. 
Likert, R. (1961), New Patterns of Management. New York: McGraw Hill.

March, J.G. and H.A. Simon (1958), Organizations. New York: John Wiley.

Maslov, S., K. Sneppen and U. Alon (2002), "Correlation Profiles and Circuit Motifs in Complex Networks," in Handbook of Graphs and Networks. John Wiley and VCH Publishers. Berlin: Wiley-Europe.

Menger, K. (1927), “Zur allgemeinen Kurventheorie,” Fundamenta Mathematicae 10, 96-115.

Moody, J. (2003), "Epidemic Potential in Human Sexual Networks: Connectivity and the Development of STD Cores." Presented at the Institute for Mathematics and its Applications, University of Minnesota, November 15-21, 2003. http://www.sociology.ohio-state.edu/jwm/presentations/ima_talk.ppt

Moody, J. (2004 forthcoming), “The structure of a Social Science Collaboration Network: Disciplinary Cohesion from 1963-1999," American Sociological Review.

Moody, J. and D.R. White (2003), "Social Cohesion and Embeddedness: A Hierarchical Conception of Social Groups," American Sociological Review, 8(1), 1-25. URL preprint: http://www.santafe.edu/ sfi/publications/Abstracts/00-08-049abs.html.

NetMiner (accessed 2004), http://www.netminer.com/NetMiner/pdf/ReferencePart1.pdf.

Newman, M.E.J. and J. Park (2003), "Why Social Networks are Different from Other Types of Networks," Phys. Rev. E, in press. http://arxiv.org/abs/cond-mat/0305612.

Newman, M.E.J., S.H. Strogatz and D.J. Watts (2001), "Random Graphs with Arbitrary Degree Distributions and their Applications," Phys. Rev. E, 64, 026118.

Owen-Smith, J., W.W. Powell and K.W. Koput (2004, forthcoming), "Pathways to an Open Elite," in J. Padgett and W.W. Powell (Eds.). Network Emergence and Transformation.

Robins, G.L., J. Woolcock and P. Pattison (in press). Small and Other Worlds: Global Network Structures from Local Processes. American Journal of Sociology.

Powell, W.W. (1990), “Neither Market nor Hierarchy: Network Forms of Organization,” Research in Organizational Behavior, 12, 295-336.

Powell, W.W. (1996), "Inter-Organizational Collaboration in the Biotechnology Industry," Journal of Institutional and Theoretical Economics, 120(1), 197-215.

Powell, W.W., and P.J. DiMaggio (1991), The New Institutionalism in Organizational Analysis. Chicago: The University of Chicago Press.

Powell, W.W., D.R. White, K.W. Koput and Jason Owen-Smith (2004, forthcoming), "Network Dynamics and Field Evolution: The Growth of Interorganizational Collaboration in the Life Sciences," American Journal of Sociology in press.

Rapoport, A. (1957), "Contribution to the Theory of Random and Biased Nets," Bulletin of Mathematical Biology, 19, 257-277.

Sewell, W.F. (1992), “A Theory of Structure: Duality, Agency, and Transformation,” American Journal of Sociology, 98, 1-29.

Simon, H. (1947), Administrative Behavior. New York, NY: Macmillan.

Snijders, T.A.B. (2001), “The Statistical Evaluation of Social Network Dynamics,” in M.E. Sobel and M.P. Becker (Eds.). Sociological Methodology 2001, Boston and London: Basil Blackwell, pp. 361-395.

Stinchcombe, A. (1990), Information and Organizations. Berkeley: University of California Press.

Uzzi, B. (1996), "The Sources and Consequences of Embeddedness for the Economic Performance of Organizations: The Network Effect," American Sociological Review, 61, 674-698.

Weber, M. (1968), Economy and Society. G. Roth and C. Wittich (Eds.). New York: The Citadel Press.

White, H.C. (2002), Markets into Networks. Princeton, NJ: Princeton University Press.

White, D.R. and F. Harary (2001), "The Cohesiveness of Blocks in Social Networks: Node Connectivity and Conditional Density," Sociological Methodology, 31(1), 305-359. Blackwell Publishers, Inc., Boston, USA and Oxford, UK. http://www.blackwellpublishing.com/abstract.asp?ref=0081$1750 \&$ vid $=31 \&$ iid $=1 \&$ aid $=98 \& \mathrm{~s}=\&$ site $=1$

White, D.R. and M. Houseman (2002), "The Navigability of Strong Ties: Small Worlds, Tie Strength and Network Topology," Complexity, 8(1), 72-81, http://eclectic.ss.uci.edu/ drwhite/Complexity.

White, D.R. and U.C. Johansen (2004), Network Analysis and Ethnographic Problems: Process Models of a Turkish Nomad Clan. Boston: Lexington Press. 
Douglas R. White a UC Irvine Professor in Anthropology and the Social Dynamics and Evolution Ph.D. program of the Institute for Mathematical Behavioral Sciences and former director of the Social Network Ph.D. program. $\mathrm{He}$ is a von Humboldt senior stiftung recipient (Distinguished Senior U.S. Social Scientist) and serves on the External Faculties of the Santa Fe Institute and the Ecoles des Hautes Etudes in Sciences Sociales. His current work focuses on mathematical anthropology, evolutionary complexity, network dynamics, and self-organizing systems.

Jason Owen-Smith is an Assistant Professor of Sociology and Organizational Studies at the University of Michigan. His work in Economic Sociology and Science and Technology Studies focuses on proccesses of organizational and institutional change in the context of academic research commercialization. Other work focuses on the dynamics of collaboration, innovation, and regional development using inter-organizational networks in research-intensive industries such as biotechnology.

James Moody is an Assistant Professor of Sociology at the Ohio State University. His research focuses broadly on social networks, with particular interest in linking individual action to global network dynamics. In addition to work on network methods, he has worked on questions related to the formation and dynamics of adolescent friendship networks, the diffusion of sexually transmitted diseases, and the network foundations for social solidarity.

Walter W. Powell is Professor of Education and affiliated Professor of Sociology and Organizational Behavior at Stanford University, and an external faculty member at the Santa Fe Institute. He works in the areas of organization theory, economic sociology, and science and technology studies. 